\title{
Importância da capacitação dos profisssionais de saúde da atenção primária: a doença renal crônica em pauta
}

Daniele Francine Pereira, Lucas Saraiva da Silva, Mário Renato Grillo Lage, Luciana Saraiva da Silva, Rosângela Minardi Mitre Cotta

\begin{abstract}
Resumo
A prevalência de doença renal crônica (DRC) vem aumentando mundialmente, tendo como agravante, o fato de ser uma enfermidade desconhecida por muitos portadores. As diretrizes clínicas recomendam identificar os indivíduos de risco, como os portadores de hipertensão arterial (HA) e diabetes mellitus (DM) e avalia-los anualmente quanto à função renal. Os profissionais que atuam na Atenção Primária à Saúde (APS) possuem papel estratégico no diagnóstico precoce e encaminhamento dos pacientes aos especialistas. No entanto, a DRC tem sido subdiagnosticada e tratada inadequadamente, resultando em maior morbimortalidade, custos ao sistema de saúde e qualidade de vida comprometida. O objetivo do estudo foi identificar a prevalência de DRC oculta em portadores de HAS, com ênfase no papel estratégico da APS na detecção precoce e no manejo adequado da DRC. Trata-se de um estudo transversal com os portadores de HAS acompanhados pela APS de um município do interior mineiro, em 2012 e 2013. Participaram do estudo 293 indivíduos. Realizaram-se entrevistas, avaliações bioquímica e antropométrica. Para o diagnóstico de DRC, utilizou-se a fórmula CKD-EPI, que é atualmente recomendada pelo Ministério da Saúde. A maioria dos indivíduos avaliados era do sexo feminino, idosos, com baixa renda, baixa escolaridade e excesso de peso. Encontrou-se prevalência de DRC oculta de 38,6\% (IC 95\%: 33,044,2), sendo que, aproximadamente $14 \%$ estavam em estágio avançado da doença. Tal resultado evidencia a necessidade de capacitação dos profissionais de saúde para superar a elevada prevalência de DRC e a problemática do diagnóstico precoce da doença em nível de APS. Os resultados sugerem que o atendimento da APS tem falhado, visto a elevada prevalência de DRC oculta nos portadores de HAS, que deveriam ser acompanhados rotineiramente. Enfatiza-se a necessidade de capacitação dos profissionais da APS, especialmente os médicos para solicitação dos exames, análise e prescrição de condutas terapêuticas adequadas, bem como de uma equipe multiprofissional integrada, a fim de manter uma efetiva continuidade do cuidado.
\end{abstract}

Descritores: Atenção Primária à Saúde; Capacitação profissional; Doença Renal Crônica. 